\title{
CROSS-PLATFORM DEVELOPMENT FOR E-COMMERCE
}

\author{
Leandro Vieira, Anabela Bernardino and Eugénia Bernardino \\ Computer Science and Communication Research Center (CIIC) \\ School of Technology and Management, Polytechnic Institute of Leiria, Portugal
}

\begin{abstract}
Electronic commerce is generally seen as a competitor for physical stores, however, this paper proposes a solution where they work side-by-side to improve the in-store customer experience. An application directed for store retailers was created with the cross-platform framework React Native, making it available for Android, iOS, and the web. Using the application, retailers can automatically view the customer's previous orders, show them products that may be of their interest, and place orders, providing the customer with a unique and faster shopping experience.
\end{abstract}

\section{KEYWORDS}

Cross-Platform, E-Commerce, Mobile, React-Native, Redux, CS-Cart

\section{INTRODUCTION}

When the majority of people think about electronic commerce or e-commerce, they think about online shopping. Although they are not incorrect, the concept of e-commerce can be a lot broader, covering other topics like digital marketing which uses the user's search history or recent purchases to show products that the customer might be interested in purchasing.

Even though e-commerce applications are a threat to real-life shops, this paper proposes an e-commerce solution that works with them side-by-side. This application would allow a retailer in a store or kiosk, to show existing products to a customer, verify stocks, create orders, and view previous orders, to name a few. The main goal of this application is to improve the customer's in-store experience, by reducing the time spent in the store while offering a more personalized visit.

This application is compatible with all major mobile operating systems (Android and iOS) and is available for the rest of the devices through the web browser. It gets the catalog and all the customer's data from an e-commerce platform developed with CS-Cart, and all operations done with a certain customer will be automatically associated with their account, meaning that they can easily visualize their information at home.

This paper is organized with a summary of the whole development process of the aplication (Section 2) and ends with the conclusion (Section 3).

\section{DEVELOPED WORK}

The goal of this project was to build an e-commerce application that worked across multiple platforms - iOS, Android, and web. The Sales Application's (SA) target audience are retailers, also known as hostesses, who work in shops or kiosks and interact with customers who walk in and are interested in buying a product or just want to know more information. This application lets the retailer have immediate access to information about the products, as well as customer information like their previously placed orders allowing for a better in-store customer experience.

For development purposes, an online technology retailer that focuses on smartphones was created. This application gathers data from CS-Cart and will be able to do some tasks that are available through CS-Cart's backend, however in a less cluttered and more straight-forward manner. 
The main functionalities provided by this application include authentication, registering or searching for an existing customer, viewing customer information (name, email, etc.), viewing products, adding or removing products to the cart, and creating, viewing, or refunding orders.

\subsection{Technologies Used}

In the following sections, we describe the technologies that were used to develop the SA.

\subsubsection{CS-Cart}

CS-Cart (CS-Cart, n.d.) was the e-commerce platform that was used in this project. This platform was the one who SA communicated with and was responsible for processing all the data and providing the Application Programming Interfaces (APIs) necessary for retrieving that data.

\subsubsection{React Native}

React Native (React Native, n.d.) was the framework used to create the application. It was created by Facebook in 2015. It uses React (Facebook n.d.), which is a JavaScript library used to build user interfaces. With React Native, it is possible to compile the same application for Android and iOS, also having the possibility of compiling it for the web, making them compatible with all devices that have a browser. Popular applications like Instagram, Facebook, and Uber were created with this framework.

\subsubsection{Redux}

In React Native, each component can have its state where their properties are stored. Although it is possible to pass down values to other components through props, that would mean that two different components that would need access to the same data would have to be associated in some form. For this reason, the component's state is ideal if the data is short-term, like for instance text in a form. For data that will stay persistent while the user navigates the application, it is best to save the data with Redux, which is a pattern for managing the state of our application (Hoffman, 2017).

With Redux (Redux, n.d.)., the entire application would have one store which would hold the entire application's state tree. This means that all the components would be able to access this store and retrieve the necessary information. The state can be manipulated through actions, which are objects that tell the store what happened. The state will never be manipulated directly by components, only with actions. The store and action are joined by a reducer, which is a function that takes the previous state and action and returns the next state to the component. Redux works with JS meaning that it is compatible with frameworks like Angular or React (Redux, n.d.).

\subsection{Architecture}

SA's architecture (see Figure 1) can be divided into two major components - Application and Redux. The application component is responsible for managing everything that the user will visualize and interact with, including the user interface components and the navigation containers. Redux has the responsibility of storing and managing all of the application's data.

The application component has two containers - the bottom bar navigation and the drawer navigation, which are subscribed to the redux store. When this store is updated, these containers are informed and will pass down the necessary information to the UI components using properties. Each component can define which property they are interested in, meaning that they will not receive unnecessary information. Once the property is received, the component can show it to the user.

The data stored in Redux can be manipulated by the UI components using actions. Once an action is called, the payload of this action is passed down to the reducer which will perform that action and update the store. To better organize the data structure, the reducer has been split into multiple reducers, being each responsible to manage a certain data structure for the application - Cart, Products, Users, Languages, Companies, Orders, Devices, and Configs. 


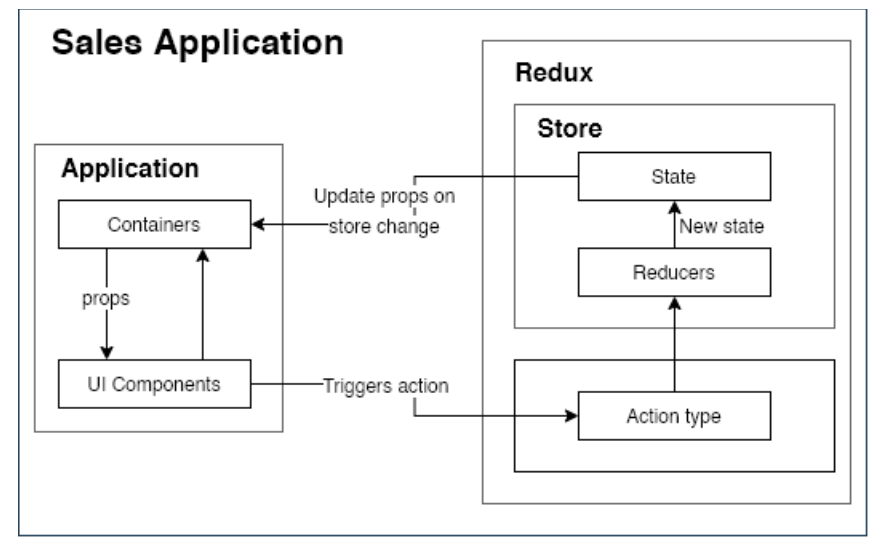

Figure 1. Application architecture

\subsection{Sales Application}

Before beginning the implementation phase of the SA, several mockups were designed so that it would be possible to gather some user feedback regarding the look and feel of the application. This allowed us to make tweaks in the user interface without having to do any coding. This also facilitated the implementation process since the design was already taken care of and it was only necessary to copy from the mockups (see Figure 2).
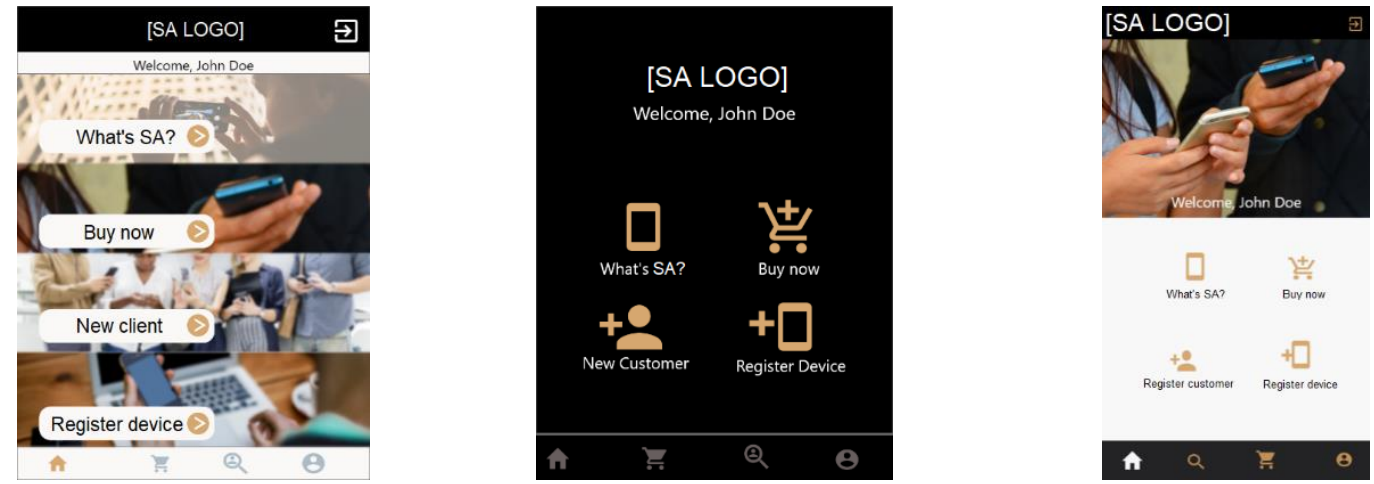

Figure 2. Evolution of the mockups (oldest to most recent)

A release-based iteration workflow was decided for the SA because time was not a crucial factor for the development, however, features were. This means that a set of features would be needed for a certain release to be complete. There were three releases, and between each release, there would be acceptance tests with users to be able to gather their input regarding how the application functioned and verify any eventual problem that they could have. This allowed improving the application for the next release. The goal of the first release was to have an application with mock data with some screens to be able to gather feedback regarding the design of the application, as well as the navigation between the screens. The second release would take that input and change accordingly, as well as get rid of all the mock data and start to use information from the CS-Cart APIs. Finally, the third release took all the suggestions from the previous release and added missing functionality, such as browser support.

Expo SDK assisted the development, build and deploy of the SA. It provided an effortless way to test the SA throughout multiple platforms through the form of reading a QR code through the Expo application from Google Play or the App Store. Normally, to compile onto an iPhone, a computer running Mac OS X with XCode would be necessary, but Expo allows to compile without any of that, giving a big advantage to those who do not own Apple computers. 
Even though the application is meant to work across multiple platforms, it does not mean that it should look exactly alike. Users from each platform are used to a specific way of how applications look and function inside their operating system, so the application must respect the design guidelines of each platform Apple's Human Interface Guidelines for iOS (Apple, no date) and Google's Material Design for Android (Google Developers, no date). This guarantees that when users use the application, they know how to use it because it is familiar. Since this application is meant to run on many types of devices, it should not only be able to adapt itself to several operating systems but to different screen sizes as well. This is also especially important because using a mobile device with a small touch screen is entirely different from using a desktop computer with a mouse and keyboard. For this reason, when the application is used on small screens, it features a bottom bar navigation which is more appropriate for touchscreens, while on devices with big screens, a sidebar navigation is used.

Some third-party components created by other developers and shared on NPM were used alongside those provided by React Native. This allowed for faster development as many components could be reused instead of needing to create one exactly alike. Not all components are compatible with all the platforms, so this must be taken into consideration once deciding on a component to use. It was not always possible to find a component with full compatibility, which was the case with the date-time picker that was not compatible with the web version.

To conclude this topic about the development, some of CS-Cart's characteristics are the possibility of having multiple stores and the addition of functionality through add-ons. Through the SA, it is possible to change the store that is currently being used, which in consequence will change which configurations are being received from the add-on. This allows for the application to be more customizable to a specific store, for example, showing a different list of products or change the default language the application uses.

\subsection{Results}

From the beginning, the goal was to create a cross-platform application that was intuitive, easy to use, and easy to understand even to someone who has never used something of the sort. Only having the developer's feedback that it meets all the requirements is not recommended, as they know all the walkarounds and the navigation surrounding the application. For this reason, it was important and helpful to have acceptance tests with users. The tests for the first release were done in person by giving the tester a device with the application, asking them to do several tasks, and study certain behaviors (if they did unnecessary taps, the time to complete the task, and if they look confused). Due to the pandemic, it was not possible to do the testing of the second release in person. As a solution, a voluntary quiz was sent by email that featured screen capture videos of a certain task being completed alongside statements relative to that task (for example, 'it was easy to complete'), and the tester would have to evaluate the statement from a scale of 1 (highly disagree) to 5 (fully agree).

Their feedback allowed for continuous improvement throughout releases, creating a better overall user experience. The testers were almost all familiar with CS-Cart and how some of the application's features were done on the backend, and there was general praise on how easy it was to complete certain tasks that were very bothersome on the backend.

In the beginning, Expo seemed like a great tool to simplify the development process and it is recommended by React Native with emphasis on it being a free service. It is free, however, when trying to create a build of the application, this build will be done on one of their cloud servers and the duration of the compilation will depend on the size of the queue and how many people are trying to compile their applications. For the process to be quicker, Expo requires a monthly payment of 29 US dollars (as of June 2020). When the first release of the application was compiled, it took over one hour to finish and the project was made public for everyone to see, having to manually switch it to private. If something goes wrong with the application, an update should be released as soon as possible and not be dependent on external factors that are not controllable by anyone in the team.

Expo also created some issues when trying to update components, as the new versions were not compatible with the respective version of Expo (for instance "react-native-community/react-native-picker" not being compatible with Expo 37). When compiling the application, Expo also warned that some of the project's dependencies were not compatible with the currently installed package version. However, when the correct version of the dependency was installed (the version Expo recommended), some components stopped working so it was better to just ignore the warnings, which might be dangerous. 
Finally, another big challenge was to try to find components that worked across all platforms. At the time of development, web compatibility was still in beta making it more difficult to find compatible components. In the case of the date picker, it was exceedingly difficult to find a component available on the web. Since React Native is based on React, there was an attempt to use a React date picker component and it worked perfectly on the web, however, even though Android or iOS were not using that specific component, it was not possible to run the application on those platforms. This means that it is only possible to use React components with React Native only if a web application is being built.

\section{CONCLUSION}

To conclude, it was possible to create an e-commerce cross-platform application. The application was tested on devices running Android 9/10 and iOS 13 (and their respective browsers), as well as browsers running on Ubuntu 18.04 and Windows 10. Creating a cross-platform application allowed for a more cost-effective way of creating an application because since it was only necessary to learn one framework and use it to have the SA available for all types of devices. The availability is especially important because depending on the country, a certain operating system might have a bigger market share than the competitor, so if you want to reach the highest number of people possible with your product, it must be available on that platform.

Due to community hype and personal curiosity around it, the React library was the one chosen for development. With this choice, there were two options for frameworks to use - React Native and Ionic. Two sample applications to manage contacts were created to get a better understanding of how it was to program in each framework, and since React was in beta in Ionic, React Native was chosen.

The objective of this project was accomplished, as the Sales Application resulted in an application available and tested on multiple platforms, with a user-friendly and modern interface. As future work, a choice would be to eliminate Expo and its dependencies from the project as they are extremely helpful in the beginning phase of the implementation but can be harmful in the long run.

\section{ACKNOWLEDGMENT}

This work was supported by national funds through the Portuguese Foundation for Science and Technology (FCT) under the project UIDB/04524/2020.

\section{REFERENCES}

Apple (no date) Human Interface Guidelines. Available at: https://developer.apple.com/design/human-interfaceguidelines/ (Accessed: 19 June 2020).

CS-Cart (no date) CS-Cart Multi-Vendor Marketplace Platform \&amp; Shopping Cart Software - Professional Ecommerce Tools. Available at: https://www.cs-cart.com/ (Accessed: 19 June 2020).

Facebook (no date) React - A JavaScript library for building user interfaces. Available at: https://reactjs.org/ (Accessed: 19 June 2020).

Google Developers (no date) Design for Android. Available at: https://developer.android.com/design (Accessed: 19 June 2020).

Hoffman, T. (2017) React State vs. Redux State: When and Why? Available at: https://spin.atomicobject.com/2017/06/07/react-state-vs-redux-state/ (Accessed: 19 June 2020).

React Native - A framework for building native apps using React (no date). Available at: https://facebook.github.io/reactnative/docs/getting-started (Accessed: 13 January 2019).

Redux (no date a) Glossary. Available at: https://redux.js.org/glossary\#state (Accessed: 19 June 2020).

Redux (no date b) Three Principles. Available at: https://redux.js.org/introduction/three-principles (Accessed: 19 June 2020). 